\title{
Absceso hepático, bacteriemia y meningoencefalitis por Klebsiella pneumoniae hipervirulenta en el curso de infección por SARS - CoV2. Reporte de caso
}

Liver abscess, bacteremia and meningoencephalitis due to an hypervirulent Klebsiella pneumonia in a patient with COVID-19. A case report

\author{
Jaime Zegarra Piérola a, a; 2 , Diana Fernández Merjildo 1, a; 2 (D), Samuel Cosme Avila ${ }^{1, \text { a }}$ (D), \\ José Ticona Salazar ${ }^{2, b}$ iD
}

\section{RESUMEN}

Klebsiella pneumoniae es miembro de la familia de enterobacterias, es un patógeno oportunista que afecta a las personas con compromiso inmune, y es causa de infecciones nosocomiales. Los serotipos capsulares K1 y K2 de Klebsiella pneumoniae tienen la característica de hipermucoviscosa, que es considerada una cepa hipervirulenta. Se presenta el caso de una mujer con absceso hepático, bacteriemia y meningoencefalitis por Klebsiella pneumoniae hipervirulenta, en el curso de una infección por SARS - COV2, quien requirió soporte ventilatorio, vasopresor, tratamiento antibiótico y drenaje percutáneo del absceso en la Unidad de cuidados intensivos del Hospital Cayetano Heredia, logrando sobrevivencia y alta hospitalaria. Este caso nos permite resaltar la presencia de la cepa hipervirulenta de Klebsiella pneumoniae en nuestro país, lo cual nos permitirá realizar una evaluación integral oportuna y tratamiento precoz.

PALABRAS CLAVE: Absceso hepático, Klebsiella pneumoniae, virulencia, meningoencefalitis. (Fuente: DeCS BIREME).

\section{SUMMARY}

Klebsiella pneumoniae $(\mathrm{Kp})$ is a member of the Enterobacteriaceae family, it is an opportunistic pathogen that affects immunosuppressed individuals. K1 and K2 strains are hyper mucous that is considered an hypervirulent factor. We report a case of a female patient who developed a hepatic abscess, bacteremia and meningoencephalitis due to an hypervirulent $\mathrm{Kp}$ strain requiring mechanical ventilation, vasopressors, antibiotic treatment and percutaneous drainage of the liver abscess in the intensive care unit at Hospital Cayetano Heredia. The patient survived and was discharge from the hospital. This case illustrates the presence of an hypervirulent Kp strain in Peru.

KEYWORDS: Liver abscess, Klebsiella pneumonia, virulence, meningoencephalitis. (Source: DeCS BIREME).

\footnotetext{
Hospital Cayetano Heredia. Lima, Perú

Universidad Peruana Cayetano Heredia. Lima, Perú

Médico Asistente de la Unidad de Cuidados Intensivos

Médico Residente de Medicina Intensiva
} 


\section{INTRODUCCIÓN}

Klebsiella es un bacilo gram negativo descubierto en 1882 por Friendler ${ }^{(1)}$, llevando su nombre desde ese entonces. Existen 7 serotipos de Klebsiella que afectan a humanos y animales. En el humano están las especies: Klebsiella pneumoniae, Klebsiella oxytoca, Klebsiella rinoescleromatis. Siendo Klebsiella peumoniae el agente más frecuente, causante de infecciones domiciliarias e intrahospitalarias ${ }^{(1,2)}$. Esta última caracterizada por presentar plásmidos productores de betalactamasa de espectro extendido y carbapenemasas, que le confieren resistencia antimicrobiana; considerando ser una causa de morbilidad y mortalidad en pacientes críticos ${ }^{(3)}$.

Desde los años de 1980 en adelante en el continente asiático se describió la presencia de una cepa de Klebsiella considerada hipervirulenta, de serotipo K1 y K2, siendo la primera más frecuente. La misma es de presentación domiciliaria, en pacientes sin factores de riesgo conocido y que cursaban con absceso hepático e invasión secundaria de otros órganos y sistemas, bacteriemia, endoftalmitis, meningitis, absceso esplénico ${ }^{(3,4)}$. Se considera una infección emergente, con extensión a diferentes países del mundo. Este agente infeccioso usualmente se caracteriza por ser sensible a los diferentes antibióticos; sin embargo, recientemente han descrito especies de Klebsiella hipervirulenta con presencia de plásmidos tipo carbapenemasa. Asimismo, existen casos reportados en pacientes con infección por SARS - CoV2 ${ }^{(5,6)}$.

Se presenta el caso de una mujer con absceso hepático, bacteriemia y meningoencefalitis por Klebsiella pneumoniae hipervirulenta, en el curso de una infección por SARS - CoV2, quien requirió soporte ventilatorio, vasopresor, tratamiento antibiótico y drenaje percutáneo del absceso en la unidad de cuidados intensivos (UCI) del Hospital Cayetano Heredia, logrando sobrevivencia y alta hospitalaria. El objetivo de presentar el presente caso fue resaltar la gravedad de la infección invasora por Klebsiella pneumoniae, y las estrategias terapéuticas al respecto.

\section{PRESENTACIÓN DEL CASO}

Mujer de 53 años, con antecedente de hipertensión arterial y diabetes mellitus tipo 2, ingresó a la emergencia del Hospital Cayetano Heredia con tiempo de enfermedad de dos semanas, caracterizado por vómitos, diarrea, dolor abdominal, alza térmica para lo cual recibió tratamiento sintomático sin mejoría; 5 horas antes del ingreso presentó debilidad en el hemicuerpo derecho y convulsiones tónicogeneralizadas. Al ingreso tenía PA $110 / 80 \mathrm{~mm} \mathrm{Hg}$, FC: $103 \times$ min; FR $24 \times$ min, $\mathrm{T} 37,5^{\circ} \mathrm{C}$, Sat $0,25 \%$ a $\mathrm{FiO}_{2} 21 \%$, Glasgow 13, rigidez nuca $(+)$, hemiparesia derecha y signo de Babinski derecho (+). A las 12 horas

Tabla 1. Parámetros clínicos, hemodinámicos y ventilatorios.

\begin{tabular}{lccc}
\hline Parámetro & Emergencia & UCI & ALTA \\
\hline PAM (mm Hg) & 70 & 80 & 70 \\
FC x min & 103 & 90 & 70 \\
Sat0 $_{2}(\%)$ & 95 & 95 & 97 \\
Lactato (mmol/l) & 2,1 & 3,2 & 0,9 \\
Mecánica Ventilatoria/Gasometría & & & \\
Volumen tidal espirado (ml) & 400 & 380 & \\
PEEP (cm de agua) & 10 & 10 & \\
Presión plateau & 22 & 20 & \\
Driving pressure & 12 & 10 & \\
Compliance estática & & & \\
$\mathrm{pH}^{\mathrm{HCO}_{3}^{-}}$ & 7,35 & 7,11 & 7,38 \\
$\mathrm{~Pa}_{2} / \mathrm{Fi}_{2}$ & 20 & 18 & 24 \\
\hline
\end{tabular}


Tabla 2. Exámenes de laboratorio.

\begin{tabular}{lcc}
\hline Parámetro & Emergencia & UCI \\
\hline Hemograma x mm & 29590 & 24580 \\
Glucosa (mg/dl) & 446 & 291 \\
Creatinina (mg/dl) & 0,7 & 0,8 \\
Hemoglobina glicosilada (\%) & & 12 \\
Presión apertura (cm de agua) & 30 & \\
Leucocitos & 700 & \\
Mononucleares (\%) & 70 & \\
Polimorfonucleares (\%) & 30 & \\
Proteínas (gr/l) & 500 & \\
Glucosa (mg/dl) & 10 \\
Tinción Gram & Gram (-) \\
\hline
\end{tabular}

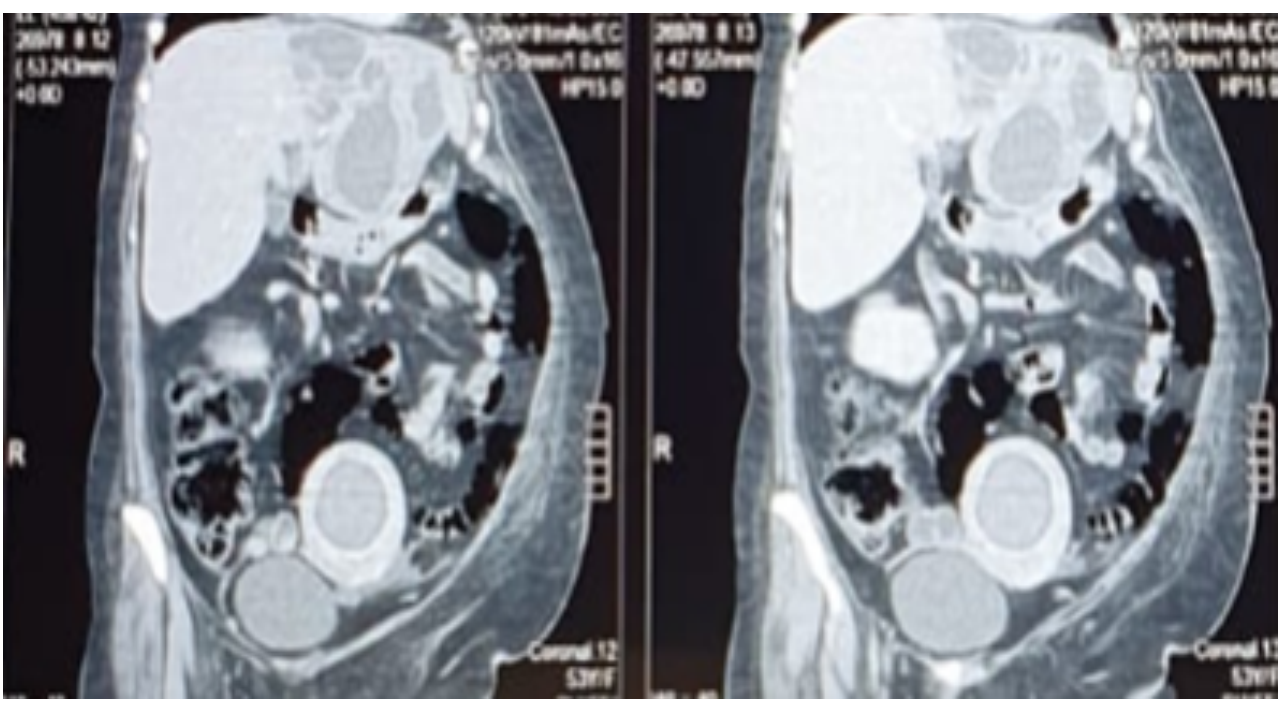

Figura 1. Tomografía espiral multicorte de abdomen. Muestra colección heterogénea multifocal en el lóbulo izquierdo del hígado (flechas).

del ingreso el Glasgow era 7, por lo cual se realizó intubación endotraqueal y ventilación mecánica invasiva.

La radiografía del tórax y la tomografía espiral multicorte (TEM) de encéfalo al ingreso no mostraron alteraciones; la punción lumbar mostró líquido cefalorraquídeo (LCR) turbio, con presión apertura de $30 \mathrm{~cm} \mathrm{H}_{2} 0$, leucocitos $700 \mathrm{x} \mathrm{mm}^{3}$, con linfomononucleares $70 \%$, polimorfonucleares $30 \%$, hematíes $150 \mathrm{x} \mathrm{mm}^{3}$, proteínas $500 \mathrm{mg} / \mathrm{dl}$, glucosa $10 \mathrm{mg} / \mathrm{dl}$, presencia de bacilos Gram negativos en la coloración Gram. El hemograma mostró leucocitosis 
PA $140 / 70 \mathrm{~mm} \mathrm{Hg}, \mathrm{FC} 68 \times \mathrm{min}, \mathrm{T} 37,2^{\circ} \mathrm{C}$, Sat $0_{2}$ $95 \%$, llenado capilar $<2$ segundos, hemograma con leucocitosis $24580 \times \mathrm{mm}^{3}$ con abastonados $15 \%$, linfocitos $750 \mathrm{xmm}^{3}$, glucosa $260 \mathrm{mg} / \mathrm{dl}$, creatinina $0,8 \mathrm{mg} / \mathrm{dl}$, calcio iónico $1,01 \mathrm{mmol} / \mathrm{l}$, deshidrogenasa láctica (DHL) $421 \mathrm{U} / 1$, transaminasa pirúvica (TGP) $13 \mathrm{mg} / \mathrm{dl}$, sodio $155 \mathrm{mEq} / \mathrm{l}$, cloro $116 \mathrm{mEq} / \mathrm{l}, \mathrm{HCO}_{3}$ 18, anión GAP 21, lactato 3,2 mmol/1, $\mathrm{Pa}_{2} / \mathrm{FiO}_{2} 240$; el cultivo de LCR y hemocultivo por 2 fueron positivos a Klebsiella pneumoniae con prueba de cuerda ("string test") $7 \mathrm{~mm}$.

Recibió tratamiento antibiótico con meropenem 2 gr cada $8 \mathrm{~h}$, insulina en infusión, soporte vasopresor, ventilación mecánica protectora con volumen tidal 6 $\mathrm{ml} / \mathrm{kg}$ peso ideal, presión plateau 26 , driving pressure

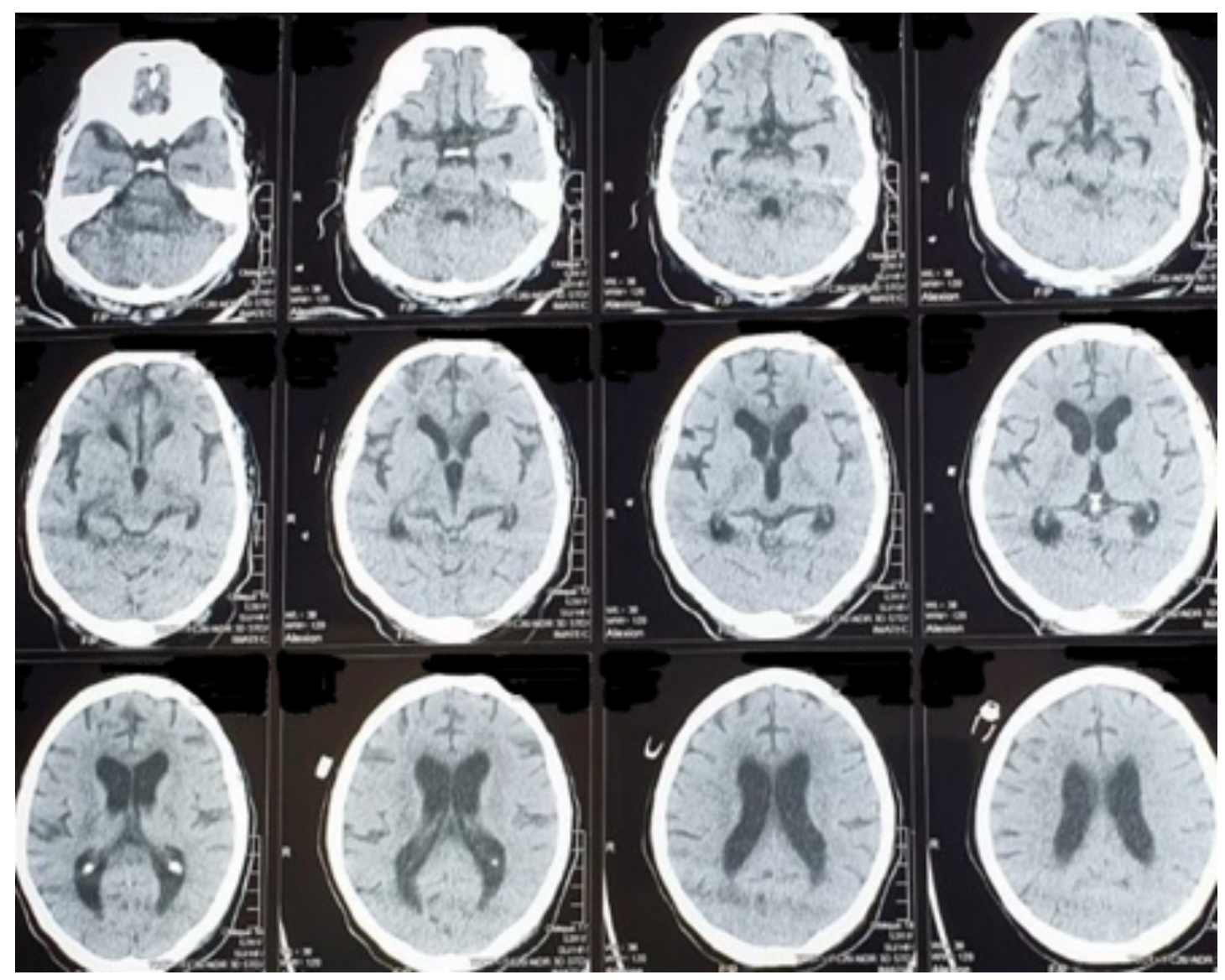

Figura 2. Tomografía espiral multicorte de Encéfalo. No evidencia de lesión.
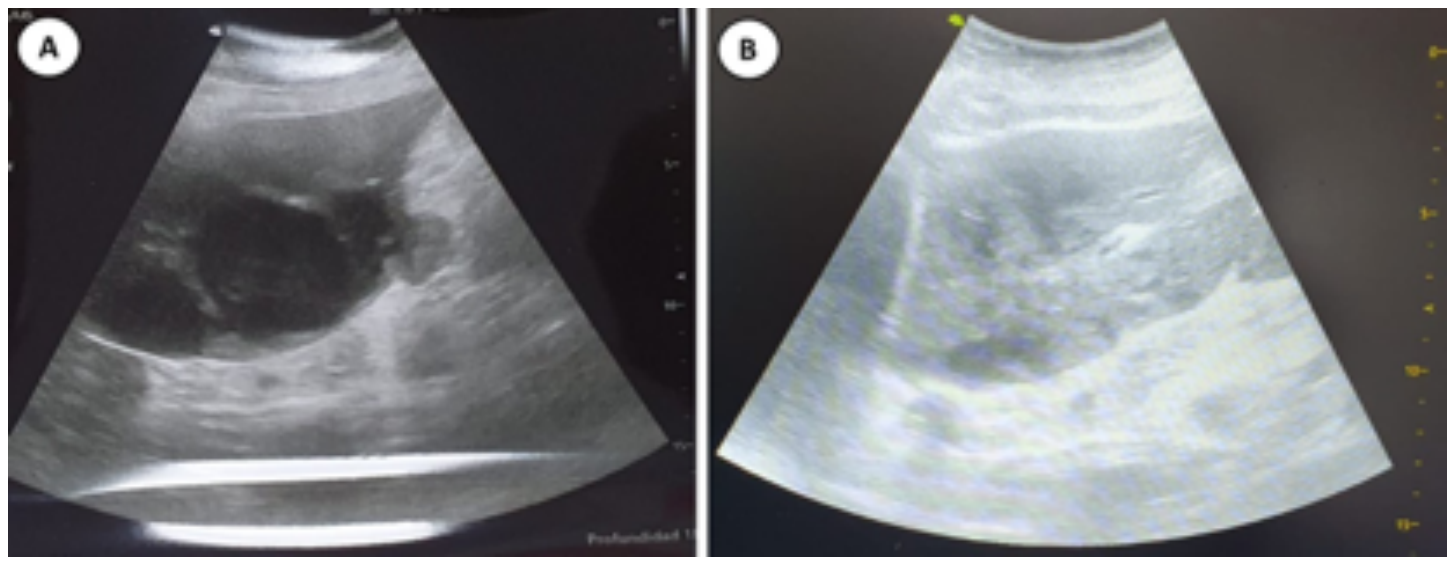

Figura 3. Ultrasonografía: Absceso hepático pre y post - drenaje percutáneo. A: absceso pre - drenaje; B: post - drenaje. 


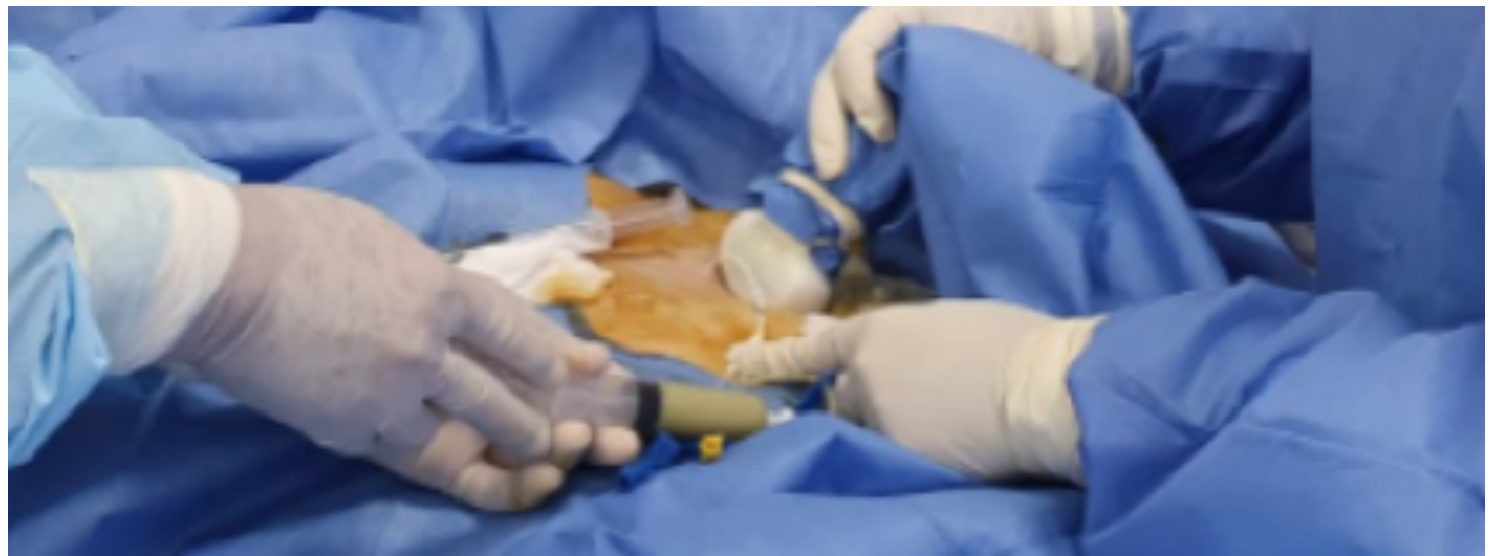

Figura 4. Drenaje percutáneo de absceso hepático con guía ecográfica, en la cama de la paciente.

12 (tabla 3). Asimismo, se realizó drenaje percutáneo del absceso hepático, a la cabecera de la paciente con guía de ultrasonografía por el personal asistencial de cuidados intensivos; se drenó $200 \mathrm{ml}$ de líquido verdoso purulento viscoso, sin complicaciones (figura 1, 3, 4). La evolución clínica fue favorable, la fiebre cedió, la leucocitosis disminuyó a menos de $10000 \mathrm{x}$ $\mathrm{mm}^{3}$; se retiró los vasopresores, se extubo, y salió alta de UCI y del hospital.

\section{DISCUSIÓN}

Se presenta el caso de una mujer con antecedente de diabetes mellitus; quien ingresó por meningoencefalitis y bacteriemia por Klebsiella pneumoniae hipervirulenta con "string test" positivo, quien cursó con deterioro neurológico, requiriendo intubación, ventilación mecánica invasiva y soporte en la UCI; y en la evaluación se identificó la presencia de un absceso hepático, considerándose el mismo como el foco de origen de la bacteriemia y meningoencefalitis.

Klebsiella pneumoniae es miembro de la familia de enterobacterias, es un patógeno oportunista que afecta a las personas con compromiso inmune, y es causa de infecciones nosocomiales ${ }^{(1,2,3)}$. Los serotipos capsulares K1 y K2 de Klebsiella pneumoniae tienen la característica de hipermucoviscosa, que es considerada una cepa hipervirulenta, y que desde el punto de vista laboratorial se identifican por una prueba de cuerda ("string test") $>5 \mathrm{~mm}$; la misma no es confirmatoria, pero si es negativa tiene un valor predictivo negativo 97\%; no existen pruebas confirmatorias, solo marcadores moleculares confiables, tipo los genes iroB, iucA, peg-344, rmpA y rmpA2 ${ }^{(4)}$.

La Klebsiella hipervirulenta es altamente invasiva afecta a personas saludables sin comorbilidades, y es causa de infección comunitaria tipo absceso hepático piógeno, bacteriemia, meningitis, fascitis necrotizante, endoftalmitis, neumonía comunitaria; siendo sensible usualmente a todos los antibióticos ${ }^{(5,6)}$. Esta variante hipervirulenta de Klebsiella pneumoniae es emergente, se describió a mediados de 1980 a 1990, en Taiwán, con un síndrome clínico de la comunidad causado por infección por Klebsiella pneumoniae ${ }^{(7)}$. Posteriormente se han descrito casos en norte América, sur América, el Caribe, Europa, medio este, Australia, África y sud África ${ }^{(7,8)}$. Asimismo, recientemente se han descrito casos de cepas hipervirulentas productoras de metalobetalactamasas, multirresistentes, de impacto en morbilidad. La paciente que reportamos tuvo una cepa sensible a todos los antibióticos.

La paciente que reportamos cursó con meningoencefalitis, con rápido deterioro neurológico. Según estudios previos, la causa más frecuente de meningoencefalitis en adultos es Streptoccoccus pneumoniae, Neisseria meningitidis, Listeria monocitogenes ${ }^{(9)}$; sin embargo, la misma con historia de diabetes mellitus descompensada como factor de riesgo, tuvo una infección comunitaria por Klebsiella pneumoniae, planteándose siembra séptica hematógena del absceso hepático. Yacuma Gutiérrez en Colombia reportó el caso de un varón sin antecedentes quien cursó con absceso hepático, bacteriemia, meningitis por Klebsiella pneumoniae ${ }^{(10)}$, de igual manera Bianca Lee et.al. ${ }^{(1)}$, en New York USA reportó el caso de un varón de 54 años con meningitis por Klebsiella pneumoniae hipervirulenta.

La diabetes mellitus es un factor de riesgo para enfermedades infecciosas debido a alteraciones en la inmunidad celular y humoral. Thomsen et al. ${ }^{(12)}$, en un estudio caso y control en Dinamarca en 1448 pacientes con absceso hepático piógeno encontró que 
los pacientes con diabetes tenían 3,6 veces más riesgo de presentar absceso hepático piógeno en comparación con los controles.

La evaluación integral nos permitió establecer el foco séptico de origen, que fue un absceso hepático piógeno, en ausencia de enfermedad hepatobiliar. Las imágenes tales como la ultrasonografía, la tomografía espiral multicorte nos permitieron establecer el diagnóstico y la primera además nos permitió realizar drenaje percutáneo a la cabecera del paciente. Meddings en su estudio poblacional de absceso hepático en EE UU, encontró que los agentes infecciosos más frecuentes fueron Streptoccoccus (29,5\%), E. coli $(18,1 \%)$, la mortalidad fue $5,6 \%$, y estuvo relacionada a edad avanzada, comorbilidades tipo cirrosis hepática, insuficiencia renal crónica, cáncer; los pacientes sometidos a drenaje percutáneo tuvieron menor mortalidad comparado con los que tuvieron drenaje quirúrgico ${ }^{(13)}$. Lederman en un reporte de 18 casos de absceso piógeno por Klebsiella en EE UU, encontró que el $83 \%$ tuvo bacteriemia concurrente y el $28 \%$ siembra hematógena ${ }^{(14)}$.

Nuestra paciente tuvo prueba antigénica positiva para SARS - CoV2, con evidencia de marcadores inflamatorios elevados, sin mayor compromiso pulmonar al ingreso. Se han reportado casos de pacientes con COVID 19 con coinfección bacteriana y nicótica; así Hosoda T. et al. ${ }^{(15)}$, en Japón reportó el caso de una mujer adulta mayor con sepsis fatal por Klebsiella hipervirulenta. Se postula a la desregulación inmune en la COVID 19, el uso de fármacos previos tipo corticosteroides, como factores de riesgo para la coinfección por Klebsiella hipervirulenta ${ }^{(15)}$.

El tratamiento incluyó soporte total en la UCI, cobertura antibiótica con Meropenem; drenaje percutáneo del absceso hepático; que fueron fundamentales en el manejo de la paciente y en la evolución clínica exitosa.

Concluimos que la paciente que reportamos cursó con infección por Klebsiella hipervirulenta, con absceso hepático, bacteriemia, meningoencefalitis en el curso de una infección por SARS - CoV2, quien sobrevivió.

\section{Declaración de conflicto de interés:}

No existe conflicto de interés en la presente publicación.

\section{Contribución de autoría:}

JZP: Participó en la concepción y redacción del artículo, recolección e interpretación de datos, así como en la aprobación de la versión final; DFM: Participó en la concepción, recolección e interpretación de datos, así como en la revisión crítica del articulo y aprobación de la versión final; SCA: Participo en la revisión del articulo y aprobación de la versión final; JTS: Participó en la recolección e interpretación de datos, así como en la revisión crítica del artículo y aprobación de la versión final.

\section{Correspondencia:}

Jaime Zegarra Piérola

Dirección electrónica: jaime.zegarra.p@upch.pe

\section{REFERENCIAS BIBLIOGRÁFICAS}

1. Pan YJ, Fang HC, Yang HC, et al. Capsular polysaccharide synthesis regions in Klebsiella pneumoniae serotype K57 and a new capsular serotype. J Clin Microbiol. 2008; 46(7): 2231-2240.

2. Pan YJ, Lin TL, Chen YH, et al. Capsular types of Klebsiella pneumoniae revisited by wzc sequencing. PLoS ONE. 2013; 8(12): e80670.

3. Hsu CR, Lin TL, Pan YJ, Hsieh PF, Wang JT. Isolation of a bacteriophage specific for a new capsular type of Klebsiella pneumoniae and characterization of its polysaccharide depolymerase. PLoS ONE. 2013; 8(8): e70092.

4. Harada S, Aoki K, Yamamoto S, et al. Clinical and molecular characteristics of Klebsiella pneumoniae isolates causing bloodstream infections in Japan: occurrence of hypervirulent infections in health care. J Clin Microbiol. 2019; 57: e01206-19. doi: 10.1128/ JCM.01206-19

5. Shon AS, Russo TA. Hypervirulent Klebsiella pneumoniae: the next superbug? Future Microbiol. 2012; 7(6):669-671.

6. Shon AS, Bajwa RP, Russo TA. Hypervirulent (hypermucoviscous) Klebsiella pneumoniae: a new and dangerous breed. Virulence. 2013; 4(2):107-118.

7. Cheng DL, Liu YC, Yen MY, Liu CY, Wang RS. Septic metastatic lesions of pyogenic liver abscess. Their association with Klebsiella pneumoniae bacteremia in diabetic patients. Arch Intern Med. 1991; 151:1557-1559. Doi: 10.1001/ archinte.1991.00400080059010

8. Wang JH, Liu YC, Lee SS, et al. Primary liver abscess due to Klebsiella pneumoniae in Taiwan. Clin Infect Dis. 1998; 26:1434-8. Doi: 10.1086/516369

9. Thigpen M. Bacterial Meningitis in the United States, 1998-2007. N Engl J Med. 2011; 364:2016-25. 
10. Yucumá-Gutiérrez S. Absceso hepático por Klebsiella pneumoniae, asociado con bacteriemia y meningitis. Reporte de un caso. Acta Med Peru. 2016; 33(1):758.

11. Lee B. Community acquired Klebsiella pneumoniae meningitis: a case report. GERMS. 2018; 8(2):93.

12. Thomsen R, Jepsen P, Sørensen H. Diabetes Mellitus and pyogenic liver abscess: Risk and Prognosis. Clin Infect Dis 2007; 44:1194-1201.

13. Meddings L, Myers RP, Hubbard J, et al. A population-based study of pyogenic liver abscesses in the United States: incidence, mortality, and temporal trends. Am J Gastroenterol. 2010; 105(1):117-24. doi: 10.1038/ajg.2009.614.
14. Lederman ER, Crum NF. Pyogenic liver abscess with a focus on Klebsiella pneumoniae as a primary pathogen: an emerging disease with unique clinical characteristics. Am J Gastroenterol. 2005; 100(2):32231. doi: 10.1111/j.1572-0241.2005.40310. x

15. Hosoda T, Harada S, Okamoto K, et al. COVID-19 y sepsis fatal causada por Klebsiella pneumoniae hipervirulenta, Japón, 2020. Emerg Infect Dis. 2021; 27 (2): 556-559. Doi: 10.3201/eid2702.204662

Recibido: 18/05/2021

Aceptado: 21/10/2021 